\title{
Sintomas internalizantes na adolescência e as relações familiares: Uma revisão sistemática da literatura
}

\author{
Adriana Raquel Binsfeld Hess - Faculdades Integradas de Taquara, Taquara/ RS, Brasil \\ Denise Falcke - Universidade do Vale do Rio dos Sinos, São Leopoldo/RS, Brasil
}

\begin{abstract}
Resumo
Este artigo teve como objetivo realizar uma revisão sistemática de literatura, nacional e internacional, no período de 2005 a 2010, sobre os sintomas internalizantes na adolescência e as relações familiares. Inicialmente, foi realizado um levantamento dos artigos indexados nas bases de dados Academic Search Premier, Education Resources Information Center (ERIC), ISI Web of Knowledge, Lilacs, MEDLINE with Full Texte SciELO (Scientific Electronic Library Online), utilizando-se os descritores, em português, "problemas internalizantes" ou "sintomas internalizantes" e "adolescência ou adolescente ou jovem" e "família"; e, em inglês, "internalizing problems or internalizing symptoms" and "adolescence or adolescent or teenager" and "family". Os artigos foram classificados de acordo com o ano de publicação, o país, a metodologia, o instrumento de mensuração para comportamentos internalizantes e, ainda, quanto à temática principal. Na literatura internacional, constatou-se um predomínio de estudos empíricos e de caráter quantitativo, realizados preponderantemente pelos Estados Unidos. No Brasil, observou-se uma grande escassez de estudos integrando as temáticas da sintomatologia internalizante na adolescência e as relações familiares. Foram encontrados somente dois artigos, o que indica a necessidade de realização de mais pesquisas nesta área em nosso país.

Palavras-chave: Adolescência, Sintomas internalizantes, Relações familiares.
\end{abstract}

Internalizing symptoms in adolescence and family relationships: a sys tematic literature review

\begin{abstract}
This article aims to perform a systematic literature review, national and international, in the period betw een 2005 to 2010 , on internalizing symptoms in adolescence and family relationships. Initially, a survey was conducted in indexed articles in the Academic Search Premier, Education Resources Information Center (ERIC), ISI Web of Knowledge, Lilacs, MEDLINE databases with Full Text, and SciELO (Scientific Electronic Library Online), using the descriptors in Portuguese, "problemas internalizantes" or "sintomas internalizantes" and "adolescência ou adolescente ou jovem" and "família" and in English, "internalizing problems or internalizing symptoms" and "adolescence or adolescent or teenager" and "family". The articles were classified according to the publication year, country, the methodology, the measurement instrument for internalizing behaviors, and also about the main issue related. In the international literature, we found a predominance of empirical studies and quantitative character, performed mainly in the United States. In Brazil, there was a paucity of studies integrating the themes of internalizing symptoms in adolescence and family relationships. We found only two articles, which indicates the need for more research in this area in our country.
\end{abstract}

Keywords: Adolescence, Internalizing symptoms, Family relations.

Síntomas de internalización en la adolescencia y las relaciones familiares: una revisión sistemática de la literatura

\begin{abstract}
Resumen
Este artículo tiene como objetivo realizar una revisión sistemática de la literatura nacional e internacional, en el período 2005-2010, a cerca de los síntomas de internalización en la adolescencia y las relaciones familiares. Al principio, se realizó un levantamiento de artículos indexados en las bases de datos Academic Search Premier, Education Resources Information Center (ERIC), ISI Web of Knowledge, Lilacs, MEDLINE with Full Text y SciELO (Scientific Electronic Library Online), utilizando los descriptores en portugués, "problemas internalizantes" o "sintomas internalizantes" y "adolescência o adolescente ojovem" " "família", y, en inglés, "internalizing problems o internalizing symptoms" y "adolescence o adolescent o teenager" y "family". Los artículos fueron clasificados de acuerdo con el año de publicación, el país, la metodología, el instrumento de medición y también cuanto a la temática principal. En la literatura internacional, se observó un predominio de estudios empíricos y de carácter cuantitativo, realizados principalmente por los Estados Unidos. En Brasil, se observó una escasez de estudios que integren las temáticas de la sintomatología de internalización de los adolescentes y las relaciones familiares. Fueron encontrados sólo dos artículos, lo que indica la necesidad de realización de más investigaciones en esta área en nuestro país.

Palabras clave: Adolescencia, Síntomas de internalización, Relaciones familiares.
\end{abstract}

A adolescência é uma fase do desenvolvimento humano que compreende mudanças físicas, sociais e psicológicas, marcada por transformações orgânicas e emocionais (Bessa, 2004). Caracteriza-se, ainda, por ser um período de instabilidade emocional, de busca da identidade, autoafirmação e independência individual, no qual há um distanciamento da família de origem e uma maior aproximação com o grupo de iguais
(Wagner, Falcke, Silveira \& Mosmann, 2002). Assim, em face dessas mudanças, a adolescência pode ser considerada um período de vulnerabilidade para o desenvolvimento de problemas emocionais e de comportamento (Jacobs, Reinecke, Gollan \& Kane, 2008).

Diversas são as definições sobre Problemas Emocionais e de Comportamento (PEC) na infância e 
adolescência, não havendo na literatura científica um consenso sobre este conceito. Segundo Achenbach (1991), os problemas emocionais e de comportamento são caracterizados por padrões sintomáticos, os quais podem ser divididos em dois tipos, denominados por ele como externalizantes e internalizantes. Os transtornos externalizantes são aqueles que se expressam em relação à outras pessoas. Referem-se a comportamentos como dificuldade em controlar impulsos, hiperatividade, agressividade e presença de raiva e delinquência. Comumente estão ligados ao transtorno da conduta e ao transtorno desafiador opositivo (Achenbach \& Howell, 1993; Associação Americana de Psiquiatria [APA], 2002). Os transtornos internalizantes são os que se expressam em relação ao próprio indivíduo. Caracterizam-se pela tristeza, retraimento, queixas somáticas e medo. Geralmente estão relacionados aos transtornos de humor (depressão) e ansiedade (Achenbach \& Howell, 1993; APA, 2002).

As Escalas ASEBA (Achenbach System of Empirically Based Assessment) foram desenvolvidas para verificar diversos aspectos de funcionamento adaptativo e desadaptativo, com base na avaliação dos problemas comportamentais e emocionais. As Escalas ASEBA são compostas por diversos instrumentos, os quais são utilizados de acordo com a faixa etária do participante. Dentre os instrumentos que compõe as escalas ASEBA, destaca-se, para o período da adolescência, o Youth Self-Report (YSR), traduzido para o Português como Inventário de Autoavaliação de Jovens de 11 a 18 anos (Rocha, Araújo \& Silvares, 2008). O YSR é utilizado para mensurar problemas emocionais e de comportamento em adolescentes, de 11 a 18 anos de idade, na visão deles próprios.

A prevalência dos problemas emocionais e de comportamento varia de cultura para cultura. Estudos em países em desenvolvimento sugerem uma taxa de $10 \%$ a $20 \%$ de prevalência em crianças e adolescentes (Hackett \& Hackett, 1999), havendo um maior nível de problemas externalizantes no sexo masculino e de internalizantes no sexo feminino (Canino e cols., 2004; Fleitlich-Bilyk \& Goodman, 2004; Hackett \& Hackett, 1999; Silva \& Hutz, 2002; Sourander, Niemela, Santalahti, Helenius \& Piha, 2008).

No Brasil, estudo epidemiológico com uma amostra de 634 crianças de quatro anos, utilizando as Escalas ASEBA, revelou uma prevalência clínica e limítrofe de $24 \%$, não apontando diferenças de gênero (Anselmi, Piccinini, Barros \& Lopes, 2004). Também usando as Escalas ASEBA, Predebon e Wagner (2005) realizaram um estudo com 523 adolescentes de 15 a 18 anos, da cidade de Porto Alegre (RS), e encontraram uma prevalência clínica de $22 \%$, mostrando altos níveis de problemas emocionais e de comportamento na amostra investigada. Convém salientar que apesar das Escalas ASEBA serem amplamente utilizadas no contexto brasileiro, algumas de suas escalas ainda estão em processo de validação no Brasil, de modo que esses resultados podem não demonstrar a real prevalência dos problemas emocionais e de comportamentos nas amostras investigadas, pelos pontos de corte terem sido estabelecidos por uma amostra americana.

Além das características individuais dos adolescentes, a estrutura familiar, os aspectos relacionais e as características dos pais podem estar vinculados ao surgimento de problemas emocionais e de comportamento (Avanci, Assis \& Oliveira, 2008; Cruvinel \& Boruchovitch, 2009; Hackett \& Hackett, 1999; Sheeber e cols., 1997; Stark, Humphrey, Crook \& Lewis, 1990; Teodoro, Cardoso \& Freitas, 2009). Segundo Silvares e Souza (2008), a familia em situação de crise pode ser considerada um fator de risco para o surgimento de PEC nos(as) filhos(as).

Diante do exposto, este estudo teve por objetivo fazer uma revisão da literatura nacional e internacional publicada no período de 2005 a 2010 sobre os sintomas internalizantes na adolescência e as relações familiares.

\section{Método}

O presente estudo foi delineado com o objetivo de realizar uma revisão sistemática da literatura, a qual utiliza métodos sistemáticos e explícitos para identificar, selecionar e avaliar pesquisas relevantes que respondam à questão de pesquisa (Khan, Kunz, Kleijnen \& Antes, 2003). Inicialmente foram realizadas buscas eletrônicas de artigos publicados de 2005 a 2010, indexados nas bases de dados do Academic Search Premier, Education Resources Information Center (ERIC), ISI Web of Knowledge, Lilacs, MEDLINE with Full Text e SciELO (Scientific Electronic Library Online). Foram utilizadas como descritores as palavras em português: "problemas internalizantes" ou "sintomas internalizantes" e "adolescência ou adolescente ou jovem" e "família" e em inglês: "internalizing problems or internalizing symptoms" and "adolescence or adolescent or teenager" and "family". A escolha desses termos justificase por estarem relacionadas ao objetivo do estudo.

Os artigos foram examinados e selecionados conforme os seguintes critérios de inclusão: disponibilidade do texto completo e estudos que abordem especificamente os transtornos internalizantes no período da adolescência e que tenham alguma relação com o aspecto familiar. Após esse levantamento inicial foram encontradas 598 publicações. 
Em seguida, foi realizada uma revisão minuciosa, sendo excluídos os artigos que não satisfaziam os critérios de inclusão. Os artigos referidos em mais de uma base de dados foram considerados apenas uma vez. Dessa forma, obteve-se um total de 162 publicações a serem analisadas. Os artigos foram classificados conforme o ano de publicação, o país de realização do estudo, a metodologia utilizada, o instrumento de mensuração para comportamentos internalizantes, e as temáticas mais abordadas.

\section{Resultados}

$\mathrm{Na}$ primeira etapa da revisão, foram encontradas 598 publicações nas bases pesquisadas, conforme pode ser observado na Tabela 1.

Tabela 1. Número de publicações de acordo com as bases de dados

\begin{tabular}{lc}
\hline \multicolumn{1}{c}{ Base de dados } & Publicações \\
\hline Academic Search Premier & 177 \\
Education Resources Information Center (ERIC) & 89 \\
ISI Web of Knowledge & 191 \\
MEDLINE with Full Text & 140 \\
Lilacs & 1 \\
SciELO (Scientific Electronic Library Online) & 0 \\
\hline Total & 598 \\
\hline
\end{tabular}

Como mencionado anteriormente, após a revisão de acordo com os critérios de inclusão e exclusão, restaram 162 artigos para análise. Com relação ao local de realização dos estudos, observa-se a predominância de pesquisas realizadas nos Estados Unidos, totalizando 63,58\% dos artigos. As demais foram desenvolvidas nos seguintes países: Holanda (14), Canadá (8), Austrália (5), Finlândia (4), Inglaterra (4), Alemanha (3), Brasil (2), Grécia (2), Noruega (2), Suécia (2), Bélgica (1), Espanha (1), Hungria (1), Israel
(1), Itália (1), Islândia (1). Seis estudos foram caracterizados como transculturais por abrangerem amostras de diferentes países.

No que tange à questão metodológica e ao ano de publicação, foram encontrados 152 estudos quantitativos e 10 artigos teóricos, havendo uma distribuição praticamente uniforme no número de artigos publicados ao longo dos últimos cinco anos, conforme mostra a Tabela 2. Não foram encontrados artigos qualitativos.

Tabela 2. Classificação metodológica dos estudos, de acordo com o ano de realização

\begin{tabular}{cccc}
\hline Ano & Quantitativos & Qualitativos & Teóricos \\
\hline 2005 & 20 & 0 & 2 \\
2006 & 32 & 0 & 0 \\
2007 & 29 & 0 & 0 \\
2008 & 30 & 0 & 4 \\
2009 & 35 & 0 & 2 \\
2010 & 6 & 0 & 2 \\
\hline Total & 152 & 0 & 10 \\
\hline
\end{tabular}

Com relação aos instrumentos de mensuração dos problemas de comportamentos internalizantes, os artigos foram categorizados de acordo com o uso ou não-uso das Escalas ASEBA, por estas serem um dos instrumentos mais utilizados mundialmente para investigar problemas emocionais e de comportamento. Para esta categorização não foram incluídos os artigos teóricos. Constatou-se que 58,21\% dos autores optaram por utilizar as escalas ASEBA para mensurar os sintomas internalizantes, confirmando a ampla utilização desses instrumentos como forma de acesso aos sintomas internalizantes dos adolescentes.

Por último, os 162 artigos foram categorizados de acordo com as temáticas mais abordadas nas publicações (vide Tabela 3). Devido à possibilidade dos artigos terem mais de um objetivo, alguns deles abrangiam mais de uma categoria temática, o que faz com que o somatório seja superior ao número de artigos examinados: 
Tabela 3. Categorias temáticas abordadas nas publicações

\begin{tabular}{lc}
\multicolumn{1}{c}{ Categoria temática } & Número de artigos \\
\hline Estrutura / contexto familiar & 50 \\
Parentalidade e conjugalidade & 48 \\
Transtornos físicos e/ ou mentais dos adolescentes & 34 \\
Característica de personalidade dos filhos & 26 \\
Eventos estressores de vida & 25 \\
Transtornos físicos e/ ou mentais dos pais & 15 \\
Intervenção terapêutica & 11 \\
Instrumentos de avaliação & 4 \\
Estudo de prevalência & 1 \\
\hline Total & 214 \\
\hline
\end{tabular}

Considerando essas categorias, serão apresentados a seguir alguns dos estudos encontrados na revisão de literatura, conforme o conteúdo que abordaram. Alguns estudos serão descritos mais detalhadamente pelo fato de melhor representarem a categoria. Além disso, quando os estudos investigaram tanto os sintomas internalizantes quanto os externalizantes, os mesmos serão mencionados como problemas emocionais e de comportamento (PEC), conforme proposto por Achenbach (1991).

1) Estrutura/ contexto familiar: essa categoria abrangeu os artigos que se destinaram a verificar associações entre a sintomatologia internalizante na adolescência e diversas variáveis familiares, tais como: configuração familiar (Breivik \& Olweus, 2006; Spruijt \& Duindam, 2005; VanderValk, Spruijt, Goede, Maas \& Meeus, 2005), suporte familiar (Oliva, Jiménez \& Parra, 2009; Wight, Botticello \& Aneshensel, 2006), conflito familiar (Davis \& Epkins, 2009; Herrenkohl, Kosterman, Hawkins \& Mason, 2009; Santiago \& Wadsworth, 2009; Skeer, McCormick, Normand, Buka \& Gilman, 2009), violência familiar (Sternberg, Lamb, Guterman \& Abbott, 2006 ), estabilidade das rotinas familiares (Ivanova \& Israel, 2006), comportamento parental (Sentse, Veenstra, Lindenberg, Verhulst \& Ormel, 2009; Steinhausen, Haslimeier \& Metzke, 2007) e nível socioeconômico das famílias (Amone-P'Olak, e cols., 2009; Wight e cols., 2006). Os artigos que compuseram esta categoria abrangeram 57 estudos $(35 \%)$ dentre os pesquisados.

Um estudo longitudinal realizado com 100 adolescentes espanhóis por Oliva e cols. (2009), demonstrou que a qualidade da relação entre pais e adolescentes mostrou-se um fator protetivo para o desenvolvimento de sintomatologia externalizante, ante a experiência de eventos estressores de vida na adolescência. Contudo, esse estudo não apontou resultados significativos para os sintomas internalizantes, tendo os autores inferido que isso possa ter acontecido pela estabilidade dos sintomas internalizantes entre o primeiro e o segundo tempo de coleta de dados, e porque os sintomas internalizantes podem depender de outros fatores, não tão relacionados ao suporte social, como, por exemplo, as estratégias de regulação emocional que os adolescentes utilizam para lidar com os eventos estressores.

Ainda considerando a relação entre pais e filhos, o clima emocional também tem sido relacionado com os problemas emocionais e de comportamento. Um estudo realizado com 131 adolescentes e suas famílias, de Denver (Estados Unidos), apontou que a compreensão e a empatia dos pais em face das experiências dos filhos são fundamentais para o desenvolvimento dos mesmos (Stocker, Richmond \& Rhoades, 2007), e quando os pais são continentes às expressões de experiências emocionais negativas pelos filhos, estes apresentam menos sintomas internalizantes. Complementando estes achados, uma investigação desenvolvida por Herrenkohl e cols. (2009), com 754 adolescentes de Seattle (Estados Unidos), indicou o conflito familiar na adolescência como preditor de sintomas depressivos no início da idade adulta.

Nesse sentido, o clima e o suporte familiar mostram-se relacionados com a diminuição dos sintomas internalizantes. Corroborando essa perspectiva, um estudo realizado por Ozer (2005), com 73 adolescentes de Berkeley (Estados Unidos), demonstrou que a percepção de suporte familiar materno diminuiu os sintomas depressivos dos filhos, assim como a percepção de suporte dos irmãos teve efeito estabilizador dos sintomas depressivos nos adolescentes. Não foram encontrados efeitos protetivos no que se refere ao suporte paterno, indicando que o pai se manteve em posição mais periférica no sistema familiar.

Leve, Kim e Pears (2005) realizaram um estudo longitudinal, com 373 sujeitos (174 meninos e 163 meninas) de Eugene (Estados Unidos), e encontraram 
que os problemas internalizantes aumentaram ao longo do tempo nas meninas, enquanto nos meninos permaneceram estáveis. As variáveis medo/timidez e depressão materna foram preditoras de comportamentos internalizantes dos filhos. Disciplina severa foi preditora de sintomas internalizantes somente para os meninos, enquanto depressão materna e renda familiar baixa foi preditora do aumento de internalizantes nas meninas. Além disso, o ajustamento conjugal teve uma associação com os sintomas internalizantes de meninos e meninas, o que evidencia a interação entre as variáveis do grupo familiar e os sintomas dos adolescentes.

A estabilidade familiar, considerada como a coerência das atividades e das rotinas familiares, também teve uma associação com os baixos níveis de sintomas internalizantes em crianças no estudo realizado por Ivanova e Israel (2006), com 70 familias de Albany (Estados Unidos). Os sintomas depressivos dos pais foram associados com problemas emocionais e de comportamento somente em crianças com baixo nível de estabilidade familiar. Dessa forma, os autores concluem que a estabilidade familiar funciona como atenuante entre os sintomas depressivos dos pais e os sintomas internalizantes dos filhos.

Considerando o contexto em que as famílias estão inseridas, diversos estudos apontaram correlações positivas entre o nível socioeconômico baixo e os sintomas depressivos (Amone-P'Olak e cols., 2009; Tonge, Hughes, Pullen, Beaufoy \& Gold, 2008; Wight e cols., 2006). Além desses, Karevold, Røysamb, Ystrom e Mathiesen (2009) identificaram, por meio de um estudo longitudinal realizado com 566 adolescentes e suas famílias, de 19 regiões da Noruega, as adversidades familiares da infância como preditoras dos sintomas ansiosos e depressivos.

Adicionalmente, a configuração familiar também tem sido relacionada ao surgimento de problemas emocionais e de comportamento na adolescência. Nesse sentido, pesquisas realizadas apontaram diferenças nos problemas de comportamento em adolescentes de famílias intactas e divorciadas, sendo que os adolescentes de famílias divorciadas apresentaram maiores níveis de sintomas internalizantes e externalizantes do que adolescentes de famílias intactas (Breivik \& Olweus, 2006; Spruijt \& Duindam, 2005; VanderValk e cols., 2005). Esses dados são corroborados pelo estudo de Schenck e cols. (2009) com 133 adolescentes mexicanos residentes no estado do Arizona (Estados Unidos), que demonstrou a importância de, nas famílias reconstituídas, considerar-se não somente o relacionamento do adolescente com o padrasto, mas também com o pai biológico não-residente.
A análise dos artigos descritos neste tópico permite constatar que, de modo geral, as relações familiares possuem impacto no surgimento de problemas emocionais e de comportamento dos filhos. Assim, apontam para a importância de as intervenções psicológicas considerarem as relações familiares no tratamento de adolescentes.

2) Parentalidade e conjugalidade: vários estudos têm apontado associações entre o conflito marital, a qualidade das relações entre pais e filhos e o surgimento de problemas emocionais e de comportamento nos filhos (Amato \& Afifi, 2006; Leve e cols., 2005; Obradović \& Hipwell, 2010; Oliva e cols., 2009; Windle et. al. 2010). Nesta categoria foram agrupados os 48 estudos (29\%) que se propuseram a investigar correlações entre as dimensões da parentalidade e da conjugalidade com os sintomas internalizantes dos adolescentes.

Em um estudo realizado com 252 adolescentes de diferentes locais da Inglaterra, Shelton e Harold (2008) encontraram associações positivas entre o conflito conjugal e a autoculpabilização do adolescente, assim como com as estratégias disfuncionais de enfrentamento dos filhos. Analisando dados do National Survey of Families and Households, dos Estados Unidos, com relação a 551 famílias de crianças com 5 a 11 anos, Gerard, Krishnakumar e Buehler (2006) revelaram associação entre o conflito conjugal e os problemas internalizantes, considerando ainda que estes são mediados parcialmente pelo conflito na relação pais-filhos. Nesta mesma perspectiva, Cui, Donnellan e Conger (2007) investigaram associações entre o ajustamento marital e os problemas internalizantes e externalizantes nos filhos adolescentes, mediante uma pesquisa realizada com 451 adolescentes e suas familias, do estado de Iowa (Estados Unidos). Os resultados apontam que o conflito parental amplia as chances dos filhos desenvolverem sintomas depressivos e delinquência, ao mesmo tempo em que esses problemas dos adolescentes agravam o conflito parental ou, de forma mais abrangente, a insatisfação conjugal. Os resultados desse estudo evidenciam a circularidade das variáveis do sistema familiar, atestando que existem influências recíprocas entre elas.

Com o objetivo de verificar as associações entre o conflito conjugal, o estresse materno e os problemas emocionais e de comportamento nos adolescentes, Salafia, Gondoli e Grundy (2008) realizaram uma pesquisa com 136 mães e seus filhos, residentes em Oklahoma (Estados Unidos). Na visão materna, os resultados indicaram que o conflito conjugal media a relação entre o seu estresse e os PEC dos filhos, o que 
demonstra que as dimensões da conjugalidade e da parentalidade podem ser consideradas indissociáveis.

Enfocando mais especificamente a parentalidade, um estudo realizado por Buehler (2006), na Carolina do Norte, com 416 adolescentes e suas famílias, constatou uma associação entre o controle parental inadequado, baixos níveis de bem-estar dos pais e os sintomas internalizantes dos filhos. Na mesma direção, Anhalt e Morris (2008) verificaram uma associação entre estilos parentais caracterizados por baixos níveis de cuidado e altos níveis de proteção com altos níveis de sintomas internalizantes, em estudo realizado com 434 estudantes de Morgantown (Estados Unidos). Bordin e cols. (2009), buscando examinar a relação entre a punição física e os problemas de saúde mental infantil, realizaram um estudo com 480 crianças brasileiras, de uma cidade do interior de São Paulo. Dentre os principais achados desse estudo, salienta-se a correlação positiva entre punição física e os problemas emocionais e de comportamento dos filhos. Resultados semelhantes foram encontrados por Bender e cols. (2007), em investigação realizada com 141 adolescentes de Glen Allen (Estados Unidos).

Ainda com relação aos estilos parentais, Lengua e Kovacs (2005) apontaram a disciplina inconsistente como preditora de problemas emocionais e de comportamento dos filhos. Esses estudos demonstram que o estilo e o controle parental são dimensões que podem ser relacionadas aos sintomas internalizantes dos filhos.

Diversas variáveis têm sido apontadas como mediadoras entre a parentalidade e o desenvolvimento de sintomas internalizantes nos adolescentes. Dentre estas, os estudos encontrados apontaram: os sintomas depressivos maternos (Corona, Lefkowitz, Sigman \& Romo, 2005), o conflito mãe-adolescente (Caples \& Barrera, 2006), o conflito interparental (Kim, Jackson, Conrad \& Hunter, 2008) e a idade materna (Dahinten, Shapka \& Willms, 2007; Pogarsky, Thornberry \& Lizotte, 2006).

Por meio das pesquisas descritas nessa categoria, pode-se perceber a interdependência entre as variáveis da parentalidade, da conjugalidade e da psicopatologia na adolescência. Dessa forma, se evidencia a complexidade do fenômeno e a necessidade de um olhar abrangente aos profissionais que atuam com famílias com filhos adolescentes.

3) Característica de personalidade dos filhos: são descritos nesta categoria, estudos que têm assinalado a correlação entre o temperamento dos filhos, as relações familiares e os sintomas internalizantes (Leve e cols., 2005; Muris \& Ollendick, 2005). Foram encontrados 34 artigos (30\%) que abordavam essas temáticas.
Buscando associações longitudinais entre o temperamento dos filhos e a parentalidade, Lengua e Kovacs (2005) realizaram um estudo com 92 crianças de Seattle (Estados Unidos), que apontou a bidirecionalidade da relação entre o temperamento dos filhos e parentalidade. De acordo com esse estudo, a disciplina inconsistente pode elevar os níveis de emoções negativas em crianças, bem como a irritabilidade da criança pode favorecer a disciplina inconsistente pelos pais, mais uma vez ficando evidente a circularidade nas relações familiares.

Confirmando a associação entre as características de personalidade dos filhos e a sintomatologia internalizante na adolescência, um estudo realizado por Nelson e cols. (2008), com 813 jovens de diferentes regiões dos Estados Unidos comparou jovens tímidos e jovens não-tímidos, e concluiu que jovens tímidos possuíam maiores níveis de sintomas internalizantes, como ansiedade e depressão, do que jovens nãotímidos. Esses dados são ainda corroborados pelo estudo longitudinal desenvolvido por Karevold e cols. (2009) com 566 adolescentes e suas familias, de 19 diferentes regiões da Noruega, que apontou a timidez como preditora de sintomatologia internalizante e como um aspecto que deve ser considerado na análise da relação pais-filhos.

4) Transtornos físicos e/ ou mentais dos pais: quinze artigos $(9,26 \%)$ encontrados investigaram as relações entre a psicopatologia dos pais e os problemas emocionais e de comportamento dos filhos. As psicopatologias mais estudadas foram: sintomas depressivos maternos (Corona e cols., 2005; Foster e cols., 2008a; Foster e cols., 2008b; Van der Toorn e cols., 2010), sintomas depressivos maternos e paternos (Elgar, Mills, McGrath, Waschbusch \& Brownridge, 2007), estresse parental (Costa, Weems, Pellerin \& Dalton, 2006), comportamentos antissociais dos pais (Herndon \& Iacono, 2005), esclerose múltipla (Diareme e cols., 2006; Steck e cols., 2007), psicopatologia materna (Spell e cols., 2008) e a saúde mental dos pais de forma ampla (Self-Brown e cols., 2006).

Em um estudo realizado por Corona e cols. (2005), com 111 díades mãe-filho(a) (62 meninas e 49 meninos), os sintomas depressivos maternos estiveram relacionados com os problemas internalizantes $\mathrm{e}$ externalizantes dos filhos. Esse estudo foi realizado com mães de origem latina, residentes em diferentes cidades dos Estados Unidos. Resultados semelhantes foram encontrados por Foster e cols. (2008b) em uma investigação realizada com 114 díades mãe-filho(a), de Pittsburgh (Estados Unidos), cujos resultados apontaram correlações positivas entre os episódios 
depressivos maternos e os níveis de sintomatologia internalizante e externalizante dos filhos.

Investigando as relações entre a psicopatologia materna e a psicopatologia dos filhos, Spell e cols. (2008) encontraram, em um estudo realizado com 260 crianças e suas mães, de Nova Orleans (Estados Unidos), associações entre os sintomas do transtorno de estresse pós-traumático (TEPT) materno os sintomas internalizantes dos filhos, sendo o TEPT apontado como um fator de risco para o surgimento de PEC nos filhos. Nessa mesma direção, o comportamento antissocial dos pais foi apontado por Herndon e Iacono (2005), em uma investigação longitudinal realizada com 1.626 famílias do estado de Minnesota (Estados Unidos), como um fator de risco para o surgimento de problemas externalizantes e internalizantes nos filhos.

De modo geral, demonstram que quando os pais apresentam sintomas emocionais e de comportamento, os filhos ficam mais suscetíveis a também apresentarem alguma manifestação de problemas emocionais e de comportamento.

5) Transtornos físicos e/ ou mentais dos adolescentes: trinta e quatro artigos $(30 \%)$ verificaram associações entre os sintomas internalizantes dos adolescentes e outros transtornos, sendo eles de natureza física e/ou mentais, tais como: asma (Alati e cols., 2005; Berz, Murdock \& Mitchell, 2005; Gupta, Crawford \& Mitchell, 2006), comportamento suicida (Liu \& Tein, 2005), problemas do sono (Reid, Hong \& Wade, 2009), transplante hepático (Gritti e cols., 2006), diabetes (Naar-King, Podolski, Ellis, Frey \& Templin, 2006), anemia/doença falciforme (Barakat, Patterson, Daniel \& Dampier, 2008; Barakat, Schwartz, Simon \& Radcliffe, 2007), paralisia cerebral (Schuengel e cols., 2006), transtorno de déficit de atenção e hiperatividade (Chen, Seipp \& Johnston, 2008; Edbom, Lichtenstein, Granlund \& Larsson, 2006), transtorno bipolar (Zelkowitz e cols., 2007), síndrome de Tourette (Roessner, Becker, Banaschewski, Freeman \& Rothenberger, 2007), transtorno obsessivo-compulsivo, (Storch e cols., 2007), transtornos alimentares (Thompson-Brenner, Eddy, Satir, Boisseau \& Westen, 2008), obesidade (Olsson e cols., 2008), anorexia nervosa (PaulsonKarlsson, Engström \& Nevonen, 2009) e o uso de álcool e drogas (Gavazzi, Lim, Yarcheck, Bostic \& Scheer, 2008; Lansford e cols., 2008; Skeer e cols., 2009).

Em um estudo realizado com 1.421 adolescentes, de Chicago (Estados Unidos), Skeer e cols. (2009) investigaram a relação entre o conflito familiar na infância e o uso de substâncias na adolescência. $O$ estudo apontou uma associação significativa entre o conflito familiar e o uso de substancias na adolescência. Com o mesmo intuito de investigar associações entre o uso de substâncias ilícitas, as relações familiares e o desenvolvimento de ansiedade e depressão, os resultados encontrados por Lansford e cols. (2008), em uma investigação realizada com 585 adolescentes das cidades de Knoxville, Nashville e Bloomington (Estados Unidos) e seus pais sugerem uma comorbidade entre o uso de substâncias e os sintomas internalizantes em jovens, de modo que $66 \%$ dos jovens participantes que preencheram critérios diagnósticos para o uso de substancias também apresentaram sintomas internalizantes ou desordens de comportamento, estando elas mediadas pelas relações familiares.

6) Eventos estressores: nessa categoria são apresentados os estudos que foram realizados com o intuito de verificar associações entre os eventos estressores, as relações familiares e o surgimento de sintomas internalizantes nos adolescentes.

Em um estudo realizado com 1.362 adolescentes chineses da Província de Shandong, Liu e Tein (2005) verificaram associações entre eventos negativos de vida, psicopatologia e comportamento suicida. Os eventos de vida negativos foram associados com o aumento do risco de problemas de externalização e internalização. Esse estudo apontou, ainda, que o estresse acadêmico e os conflitos familiares foram os estressores mais relacionados ao risco de comportamento suicida nessa população, sendo que as meninas reportaram mais ideações suicidas do que os meninos $(22 \%$ vs. $17.5 \%)$.

Appleyard, Egeland, Dulmen e Sroufe (2005) sugerem, a partir de resultados de uma pesquisa realizada com 171 adolescentes do estado de Minnesota (Estados Unidos), que quanto maior o número de fatores de risco na infância, maior é a chance de predição de problemas de comportamento na adolescência. Nessa mesma direção, os achados de um estudo longitudinal realizado por Vegt, Ende, Ferdinand, Verhulst e Tiemeier (2009), com 1.984 crianças holandesas, apontam os maus-tratos infantis como eventos de vida que se constituem em um fator de vulnerabilidade para o desenvolvimento de sintomas internalizantes.

Verificando associações entre eventos de vida estressores e problemas emocionais e de comportamento, Oliva e cols. (2009), em um estudo realizado com 100 adolescentes de Sevilha (Espanha), apontaram a qualidade da relação entre pais e adolescentes como um fator protetivo em face da experiência de eventos estressores de vida e o desenvolvimento de psicopatologias. Buscando uma 
influência cronológica entre as dimensões das relações familiares, dos eventos estressores e da psicopatologia adolescente, Herrenkohl e cols., (2009) constataram que altos níveis de conflito familiar na infância foram preditores de eventos estressores, que, por sua vez, predisseram sintomas depressivos na vida adulta, em uma investigação longitudinal realizada com 754 crianças e adolescentes de Seattle (Estados Unidos). Ainda que fique difícil pensar em uma relação tão linear entre essas dimensões, a maioria dos estudos analisados evidenciam o quanto são dimensões associadas e que devem ser consideradas em conjunto.

7) Intervenção terapêutica: onze estudos (7\%) discorreram sobre práticas e enfoques terapêuticos utilizados com familias de adolescentes que apresentam sintomas internalizantes ou grupos de adolescentes.

Ressaltando a eficácia da abordagem familiar, Paulson-Karlsson e cols. (2009) realizaram um estudo piloto, na Suécia, que avaliou o tratamento familiar da anorexia nervosa de adolescentes do sexo feminino. Trinta e duas pacientes e seus pais foram avaliados antes do tratamento, aos 18 e aos 36 meses de tratamento, quanto a sintomas de transtornos alimentares, psicopatologia e clima familiar. Aos 36 meses, houve remissão total dos sintomas de transtornos alimentares em $75 \%$ das pacientes e diminuição dos sintomas de internalização. Com relação ao clima familiar, as participantes perceberam uma maior coesão familiar na última avaliação.

A avaliação dos efeitos de um programa de intervenção baseado na família, para adolescentes com sintomas internalizantes e uso de álcool, cigarro e drogas ilícitas, foi o foco de um estudo desenvolvido por Trudeau, Spoth, Randall e Azevedo (2007) com 383 adolescentes e suas famílias, da região do CentroOeste dos Estados Unidos. Os resultados desse estudo apontaram que o grupo experimental apresentou, ao final da intervenção, menores índices de sintomas internalizantes e de uso de substâncias múltiplas.

A importância da aliança terapêutica na terapia individual e familiar de adolescentes com problemas de comportamento foi destacada por Hogue, Dauber, Stambaugh, Cecero e Liddle (2006), por meio de uma investigação realizada com 100 adolescentes e suas familias, de Nova Iorque (Estados Unidos). Segundo esses autores, a aliança forte com os pais em terapia familiar auxiliou na diminuição do consumo de drogas e dos problemas de comportamento dos adolescentes.

$\mathrm{Na}$ perspectiva da abordagem cognitivocomportamental, Harrell, Mercer e DeRosier (2009) avaliaram a eficácia de um treinamento de habilidades sociais em grupo para a melhora do ajustamento social, emocional e comportamental, e das habilidades sociais de adolescentes, realizado com 74 adolescentes da Carolina do Norte (Estados Unidos). O grupo mostrou-se eficaz, tendo os participantes apresentado melhoras no autoconceito e diminuição dos sintomas internalizantes. Ainda no que se refere ao tratamento em grupo, Keiley (2007) avaliou a eficácia de um tratamento em grupo realizado com 73 adolescentes do estado de Indiana (Estados Unidos) privados de liberdade. Os resultados demonstraram uma redução dos sintomas de internalização, bem como do consumo de álcool e drogas.

8) Instrumentos de avaliação: quatro estudos encontrados se dedicaram à análise e validação de instrumentos psicométricos utilizados para avaliação do ajustamento psicossocial de crianças e adolescentes. Gupta e cols. (2006) desenvolveram um questionário breve para investigação de problemas emocionais e de comportamento em crianças asmáticas. Os autores realizaram um estudo piloto do Childhood Asthma Resiliency Questionnaire (CARQ) com 173 crianças de Calgary (Canadá) e seus pais, cujos resultados apontaram correlações significativas $(p<0,001)$ entre os escores obtidos no CARQ e na subescala de problemas internalizantes do CBCL (Child Behavior Checklist), respondido pelos pais. Esse estudo concluiu que o CARQ é um instrumento que pode auxiliar os clínicos a identificarem riscos para o desenvolvimento de sintomas internalizantes em crianças asmáticas.

Partindo de evidências de que o CBCL pode não avaliar adequadamente problemas clínicos em diversas populações, em virtude de diferenças culturais, Mano, Davies, Klein-Tasman e Adesso (2009) realizaram um estudo com o objetivo de verificar a equivalência do CBCL entre 145 pais e adolescentes afrodescendentes. Os índices de consistência interna do CBCL obtidos no estudo variaram de 0,65 a 0,88 , sendo um pouco mais baixos do que os índices obtidos na amostra normativa. Ao final, ainda que os índices tenham sido ligeiramente diferentes, considerou-se a equivalência das medidas.

Forman e Davies (2005) realizaram um estudo com 853 adolescentes da região metropolitana dos Estados Unidos que investigou as propriedades psicométricas das escalas de Segurança no Sistema Familiar (Security in the Family System-SIFS). As escalas apresentaram boa consistência interna, tendo os resultados do estudo demonstrado que a SIFS é uma ferramenta psicométrica capaz de auxiliar no desenvolvimento de modelos de análise dos processos familiares e que a segurança emocional na familia é um construto válido para identificar os padrões de apego das crianças em diferentes relacionamentos familiares. 
9) Estudo de prevalência: estudos de prevalência são importantes para justificar e demonstrar a importância da elaboração de intervenções destinadas a populações com características específicas. Nesse sentido, um estudo encontrado, realizado por Furniss, Beyer e Guggenmos (2006), investigou a prevalência dos problemas emocionais e de comportamento em 1.887 crianças em idade pré-escolar, do Norte da Alemanha. Os resultados mostraram que a prevalência de sintomas emocionais de comportamento foi de $12,4 \%$, sendo que os sintomas internalizantes foram significativamente mais frequentes do que os externalizantes.

\section{Considerações finais}

Este artigo visou traçar um panorama dos estudos que foram realizados nos últimos anos (2005 a 2010), e que estão disponíveis nas bases de dados nacionais e internacionais (Academic Search Premmier, ISI Web of Knowledge, ERIC, MEDLINE with Full Text e SciELO), que investigaram os sintomas internalizantes na adolescência e as relações familiares.

Constatou-se uma unanimidade de estudos empíricos quantitativos, possivelmente pela busca de associação entre as duas variáveis, sendo muitos deles longitudinais, realizados com o objetivo de verificar como as associações entre as variáveis vão interagindo entre a infância e a adolescência. Além dos estudos empíricos, foram encontrados artigos teóricos. O país que apresentou o maior número de investigações foi os Estados Unidos, tendo sido constatada uma escassez de estudos realizados no Brasil, o que assinala a necessidade de ampliação das investigações nessa área em nosso país. Os instrumentos preponderantemente utilizados para a identificação de sintomas internalizantes na adolescência foram os que compõem as escalas ASEBA, coerentes com a definição desse construto que foi proposta por Achenbach (1991). Por fim, uma análise dos conteúdos dos artigos revelou que as dimensões das relações familiares estiveram evidentemente relacionadas com a manifestação dos sintomas internalizantes dos adolescentes. Muitos estudos ampliaram a complexidade da compreensão da interação entre essas dimensões, analisando outras variáveis como mediadoras ou intervenientes, tais como a psicopatologia dos pais e os eventos de vida estressores.

Ao final deste estudo, foi possível compreender a importância de se considerar o adolescente inserido na família como foco de análise das intervenções psicológicas dedicadas ao tratamento dos problemas emocionais e de comportamento manifestados por eles. Como sistemicamente nada que acontece na família pode ser considerado como exclusivo de um único membro, da mesma forma a psicopatologia do adolescente deveria ser considerada como uma manifestação do sistema familiar como um todo.

\section{Referências}

Achenbach, T. M. (1991). Manual for the Child Behavior Checklist/4-18 e 1991 profile. Burlington, VT: University of Vermont.

Achenbach, T. \& Howell, C. (1993). Are American children's problems getting worse? A 13-year comparison. Journal of American Academy on Child and Adolescent Psycbiatry, 32, 1145-1154.

Alati, R., O’Callaghan, M., Najman, J. M., Williams, G. M., Bor, W. \& Lawlor, D. A. (2005). Asthma and internalizing behavior problems in adolescence: a longitudinal study. Psychosomatic Medicine, 67, 462470.

Amato, P. R. \& Afifi. T. D. (2006). Relations with parents and subjective well-being. Journal of Marriage and Family, 68, 222-235.

Amone-P'Olak, K., Burger, H., Ormel, J., Huisman, M., Verhulst, F. C. \& Oldehinkel, A. J. (2009). Socioeconomic position and mental health problems in pre- and early-adolescents. The Trails study. Social Psychiatry \& Psychiatric Epidemiology, 44, 231-238.

Anhalt, K. \& Morris, T. L. (2008). Parenting characteristics associated with anxiety and depression: a multivariate approach. Journal of Early and Intensive Behavior Intervention, 5(3), 122-137.

Anselmi, L., Piccinini, C. A., Barros, F. C. \& Lopes, R. S. (2004). Psychosocial determinants of behaviour problems in Brazilian preschool children. Journal of Child Psychology and Psychiatry, 45, 779-788.

Appleyard, K., Egeland, B., Dulmen, M. H. M. \& Sroufe, L. A. (2005). When more is not better: the role of cumulative risk in child behavior outcomes. Journal of Child Psychology and Psychiatry, 46(3), 235245.

Associação Americana de Psiquiatria (2002). Manual diagnóstico e estatístico de transtornos mentais - IV. Porto Alegre: ArtMed.

Avanci, J. Q., Assis, S. G. \& Oliveira, R. V. C. (2008). Sintomas depressivos na adolescência: estudo sobre fatores psicossociais em amostra de escolares de um município do Rio de Janeiro, Brasil. Cadernos de Saúde Pública, 24(10), 2334- 346.

Barakat, L. P., Schwartz, L. A., Simon, K. \& Radcliffe, J. (2007). Negative thinking as a coping strategy mediator of pain and internalizing symptoms in adolescents with sickle cell disease. Journal of Behavioral Medicine, 30, 199-208. 
Barakat, L. P., Patterson, C. A., Daniel, L. C. \& Dampier, C. (2008). Quality of life among adolescents with sickle cell disease: mediation of pain by internalizing symptoms and parenting stress. Health and Quality of Life Outcomes, 6(60), 1-9.

Bender, H., Allen, J. P., Mcelhaney, K. B., Antonishak, J., Moore, C. M., Kelly, H. O. \& Davise, S. M. (2007). Use of harsh physical discipline and developmental outcomes in adolescence. Development and Psychopathology, 19, 227-242.

Berz, J. B., Murdock, K. K. \& Mitchell, D. K. (2005). Children's asthma, internalizing problems, and social functioning: an urban perspective. Journal of Child and Adolescent Psychiatric Nursing, 18(4), 181197.

Bessa, M. (2004). Quando o uso de drogas ocorre junto com outros transtornos psiquiátricos. Em L. Pinsky \& M. A. Bessa (Orgs.). Adolescência e drogas. São Paulo: Contexto.

Bordin, I. A., Duarte, C. S., Peres, C. A., Nascimento, R., Curtoa, B. \& Paula, C. S. (2009). Severe physical punishment: risk of mental health problems for poor urban children in Brazil. Bulletin of World Health Organzation, 87, 336-344.

Breivik, K. \& Olweus, D. (2006). Adolescent's adjustment in four post-divorce family structures: single mother, stepfather, joint physical custody and single father families. Journal of Divorce \& Remarriage, 44(3), 99-124.

Buehler, C. (2006). Parents and peers in relation to early adolescent problem behavior. Journal of Marriage and Family, 68, 109-124.

Canino, G., Shrout, P. E, Rubio-Stipec, M., Bird, H. R., Bravo, M., Ramírez, R., Chaves, L., Alegría, M., Bauermeister, J. J., Hohmann, A., Ribera, J., García, P. \& Martínez-Taboas, A. (2004). The DSM-IV rates of child and adolescent disorders in Puerto Rico: prevalence, correlates, service use, and the effects of impairment. Archives of General Psychiatry, 61, 85-93.

Caples, H. S. \& Barrera Jr., M. (2006) Conflict, support and coping as mediators of the relation between degrading parenting and adolescent adjustment. Journal of Youth and Adolescence, 35(4), 603-615.

Chen, M., Seipp, C. M. \& Johnston, C. (2008). Mothers' and fathers' attributions and beliefs in families of girls and boys with Attention-Deficit/ Hyperactivity Disorder. Child Psychiatry Human Development, 39, 85-99.

Corona, R., Lefkowitz, E. S., Sigman, M. \& Romo, L. F. (2005). Latino adolescents' adjustment, maternal depressive symptoms, and the mother-adolescent relationship. Family Relations, 54, 386-399.
Costa, N. M., Weems, C. F., Pellerin, K. \& Dalton, R. (2006). Parenting stress and childhood psychopathology: an examination of specificity to internalizing and externalizing symptoms. Journal of Psychopathology and Behavioral Assessment, 28(2), 113122.

Cruvinel, M. \& Boruchovitch, E. (2009). Sintomas de depressão infantil e ambiente familiar. Psicologia em Pesquisa-UFJF, 3, 87-100.

Cui, M., Donnellan, M. B. \& Conger, R. D. (2007). Reciprocal influences between parents' marital problems and adolescent internalizing and externalizing behavior. Developmental Psychology, 43(6), 1544-1552.

Dahinten, V. S., Shapka, J. D. \& Willms, J. D. (2007). Adolescent children of adolescent mothers: the impact of family functioning on trajectories of development. Journal of Youth \& Adolescence, 36, 195-212.

Davis, K. A. \& Epkins, C. C. (2009). Do private religious practices moderate the relation between family conflict and preadolescents' depression and anxiety symptoms? Journal of Early Adolescence, 29(5), 693-717.

Diareme, S., Tsiantis, J., Kolaitis, G., Ferentinos, S., Tsalamanios, E., Paliokosta, E., Anasontzi, S., Lympinaki, E., Anagnostopoulos, D. C., Voumvourakis, C. \& Romer, G. (2006). Emotional and behavioural difficulties in children of parents with multiple sclerosis. A controlled study in Greece. European Child \& Adolescent Psychiatry, 15(6), 309-318.

Edbom, T., Lichtenstein, P., Granlund, M. \& Larsson, J. (2006). Long-term relationships between symptoms of attention deficit hyperactivity disorder and self-esteem in a prospective longitudinal study of twins. Acta Pediatrica, 95, 650657.

Elgar, F. J., Mills, R. S. L., McGrath, P. J., Waschbusch, D. A. \& Brownridge, D. A. (2007). Maternal and paternal depressive symptoms and child maladjustment: the mediating role of parental behavior. Journal of Abnorm Child Psychology, 35, $943-$ 955.

Fleitlich-Bilyk, B. \& Goodman, R. (2004), Prevalence of child and adolescent psychiatric disorders in southeast Brazil. Journal of American Academy Child and Adolescence Psychiatry, 43(6), 727-734.

Forman, E. M. \& Davies, P. T. (2005). Assessing children's appraisals of security in the family system: the development of the Security system: the development of the Security in the Family System (SIFS) scales. Journal of Child Psychology and Psychiatry, 46(8), 900-916. 
Foster, C. E., Webster, M. C., Weissman, M. M., Pilowsky, D. J., Wickramaratne, P. J., Rush, A. J., Hughes, C. W., Garber, J., Malloy, E., Cerda, G., Kornstein, S. G., Alpert, J. E., Wisniew ski, S. R., Trivedi, M. H., Fava, M. \& King, C. A. (2008a). Course and severity of maternal depression: associations with family functioning and child adjustment. Journal of Youth \& Adolescence, 37, 906916

Foster, C. E., Webster, M. C., Weissman, M. M., Pilowsky, D. J., Wickramaratne, P. J., Talati, A., Rush, A. J., Hughes, C. W., Garber, J., Malloy, E., Cerda, G., Kornstein, S. G., Alpert, J. E., Wisniewski, S. R. \& Trivedi, M. H. (2008b). Remission of maternal depression: relations to family functioning and youth internalizing and externalizing symptoms. Journal of Clinical Child \& Adolescent Psychology, 37(4), 714-724.

Furniss, T., Beyer, T. \& Guggenmos, J. (2006). Prevalence of behavioural and emotional problems among six-years-old preschool children: Baseline results of a prospective longitudinal study. Social Psychiatry \& Psychiatric Epidemiology, 41, 394-399.

Gavazzi, S. M., Lim, J., Yarcheck, C. M., Bostic, J. M. \& Scheer, S. D. (2008). The impact of gender and family processes on mental health and substance use issues in a sample of court-involved female and male adolescents. Journal of Youth \& Adolescence, 37, 1071-1084.

Gerard, J. M., Krishnakumar, A. \& Buehler, C. (2006). Marital conflict, parent-child relations, and youth maladjustment. A longitudinal investigation of spillover effects. Journal of Family Issues, 27(7), 951975.

Gritti, A., Sicca, F., Di Sarno, A. M., Di Cosmo, N., Vajro, S. \& Vajro, P. (2006). Emotional and behavioral problems after pediatric liver transplantation: a quantitative assessment pediatric transplantation. Pediatric Transplantation, 10, $205-$ 209.

Gupta, S., Crawford, S. G. \& Mitchell, I. (2006). Screening children with asthma for psychosocial adjustment problems: a tool for health care professionals. Journal of Asthma, 43, 543-548.

Hackett, R. \& Hackett, L. (1999). Child psychiatry across cultures. International Review of Psychiatry, 11, 225- 235.

Harrell, A. W., Mercer, S.H. \& DeRosier, M. E. (2009) Improving the social-behavioral adjustment of adolescents: the effectiveness of a social skills group intervention. Journal of Child and Family Studies, 18, 378-387.
Herndon, R. W. \& Iacono, W. G. (2005). Psychiatric disorder in the children of antisocial parents. Psychological Medicine, 35, 1815-1824.

Herrenkohl, T. I., Kosterman, R., Hawkins, J. D. \& Mason, W. A. (2009). Effects of growth in family conflict in adolescence on adult depressive symptoms: Mediating and moderating effects of stress and school bonding. Journal of Adolescent Health, 44, 146-152.

Hogue, A., Dauber, S., Stambaugh, L. F., Cecero, J. J. \& Liddle, H. A. (2006). Early therapeutic alliance and treatment outcome in individual and family therapy for adolescent behavior problems. Journal of Consulting and Clinical Psychology, 74(1), 121-129.

Ivanova, M. Y. \& Israel, A. C. (2006). Family stability as a protective factor against psychopathology for urban children receiving psychological services. Journal of Clinical Child and Adolescent Psychology, 35(4), 564-570.

Jacobs, R. H., Reinecke, M. A., Gollan, J, K. \& Kane, P. (2008). Empirical evidence of cognitive vulnerability for depression among children and adolescents: a cognitive science and developmental perspective. Clinical Psychology Review, 28, 759-782.

Karevold, E., Røysamb, E., Ystrom, E. \& Mathiesen, K. S. (2009). Predictors and pathways from infancy to symptoms of anxiety and depression in early adolescence. Developmental Psychology, 45(4), 10511060.

Keiley, M. K. (2007). Multiple-family group intervention for incarcerated adolescents and their families: a pilot project. Journal of Marital and Family Therapy, 33(1), 106-124.

Khan, K. S., Kunz, R., Kleijnen, J. \& Antes, G. (2003 Five steps to conducting a systematic review. London. Journal of the Royal Society, 96(3), 118-121.

Kim, K. L., Jackson, Y., Conrad, S. M. \& Hunter, H. L. (2008). Adolescent report of interparental conflict: the role of threat and self-blame appraisal on adaptive outcome. Journal of Child and Family Studies, 17, 735-751.

Lansford, J. E., Erath, S., Yu, T., Pettit, G. S., Dodge, K. A. \& Bates, J. E. (2008). The developmental course of illicit substance use from age 12 to 22: links with depressive, anxiety, and behavior disorders at age 18. Journal of Child Psychology and Psychiatry, 49(8), 877-885.

Lengua, L. J. \& Kovacs, E. A. (2005). Bidirectional associations between temperament and parenting and the prediction of adjustment problems in middle childhood. Applied Developmental Psychology, 26, 21-38.

Leve, L. D., Kim, H. K. \& Pears K. C. (2005). Childhood temperament and family environment 
as predictors of internalizing and externalizing trajectories from ages 5 to 17. Journal of Abnormal Child Psychology, 33(5), 505-520.

Liu, X. \& Tein, J. (2005). Life events, psychopathology, and suicidal behavior in Chinese adolescents. Journal of Affective Disorders, 86, 195-203

Mano, K. E. J., Davies, W. H., Klein-Tasman, B. P. \& Adesso, V. J. (2009). Measurement equivalence of the Child Behavior Checklist among parents of African American Adolescents. Journal of Child and Family Studies, 18, 606-620.

Muris, T. \& Ollendick, T. H. (2005). The role of temperament in the etiology of child psychopathology. Clinical Child and Family Psychology Review, 8(4), 271-289.

Naar-King, S., Podolski, C., Ellis, D. A., Frey, M. A. \& Templin, T. (2006). Social ecological model of illness management in high-risk youths with type 1 diabetes. Journal of Consulting and Clinical Psychology, 74(4), 785-789.

Nelson, L. J., Padilla-Walker, L. M., Badger, S., Barry, C. M., Carroll, J. S. \& Madsen, S. D. (2008). Associations between shyness and internalizing behaviors, externalizing behaviors, and relationships during emerging adulthood. Journal of Youth \& Adolescence, 37, 605-615.

Obradović, J. \& Hipwell, A. (2010). Psychopathology and social competence during the transition to adolescence: the role of family adversity and pubertal development. Development and Psychopathology, 22, 621-634.

Oliva, A., Jiménez, J. M. \& Parra, A. (2009). Protective effect of supportive family relationships and the influence of stressful life events on adolescent adjustment. Anxiety, Stress \& Coping, 22(2), 137152.

Olsson, G. M., Marild, S., Alm, J., Brodin, U., Rydelius, P. \& Marcus, C. (2008). The Adolescent Adjustment Profile (AAP) in comparisons of patients with obesity, phenylketonuria or neurobehavioural disorders. Nordic Journal of Psychiatry, 62(1), 66-76.

Ozer, E. J. (2005). The impact of violence on urban adolescents: longitudinal effects of perceived school connection and family support. Journal of Adolescent Research, 20(2), 167-192.

Paulson-Karlsson, G., Engström, I. \& Nevonen, L. (2009). A pilot study of a family-based treatment for adolescent anorexia nervosa: 18- and 36-month follow-ups. Eating Disorders, 17, 72-88.

Pogarsky, G., Thornberry, T. P. \& Lizotte, A. J. (2006). Developmental outcomes for children of young mothers. Journal of Marriage and Family, 68, 332-344.
Predebon, J. C. \& Wagner, A. (2005). Problemas de comportamento na adolescência: configuração familiar e aspectos sóciodemográficos. Práxis, 2(2), $\mathrm{s} / \mathrm{n}$.

Reid, G. J., Hong, R. Y. \& Wade, T. J. (2009). The relation between common sleep problems and emotional and behavioral problems among 2 - and 3 -year-olds in the context of known risk factors for psychopathology. Journal of Sleep Research, 18, 49-59.

Rocha, M. M., Araújo, L. G. S. \& Silvares, E. F. M. (2008). Um estudo comparativo entre duas traduções brasileiras do Inventário de AutoAvaliação para Jovens (YSR). Psicologia: Teoria e Prática, 10, 14-24.

Roessner, V., Becker, A., Banaschewski, T., Freeman, R. D. \& Rothenberger, A. (2007). Developmental psychopathology of children and adolescents with Tourette syndrome - impact of ADHD. European Child \& Adolescent Psychiatry, 16, 24-35.

Salafia, E. H. B., Gondoli, D. M. \& Grundy, A. M. (2008). Marital conflict as a mediator of the longitudinal connections between maternal emotional distress and early adolescent maladjustment. Journal of Child and Family Studies, 17, 928-950.

Santiago, C. D. \& Wadsworth, M. E. (2009). Coping with family conflict: What's helpful and what's not for low-income adolescents. Journal of Child and Family Studies, 18, 92-202.

Schenck, C. E., Braver, S., Wolchik, S. A., Saenz, D., Cookston, J. T. \& Fabricius, W. V. (2009). Relations between mattering to step- and nonresidential fathers and adolescent mental health. Fathering, 7(1), 70-90.

Schuengel, C., Voorman, J., Stolk, J., Dallmeijer, A., Vermeer, A. \& Becher, J. (2006). Self-worth, perceived competence, and behaviour problems in children with cerebral palsy. Disability and Rehabilitation, 28(20), 1251-1258.

Self-Brown, S. R., LeBlanc, M., Kelley, M. L., Hanson, R., Laslie, K. \& Wingate, A. (2006). Effects of community violence exposure and parental mental health on the internalizing problems of urban adolescents. Violence and Victims, 21(2), 183-198.

Sentse, M., Veenstra, R., Lindenberg, S., Verhulst, F. C. \& Ormel, J. (2009). Buffers and risks in temperament and family for early adolescent psychopathology: Generic, conditional, or domainspecific effects? Developmental Psychology, 45(2), 419430.

Sheeber, L., Hops, H., Alpert, A., Davis, B. \& Andrews, J. (1997). Family support and conflict: Prospective relations to adolescent depression. Journal of Child Psychology, 25, 333-344. 
Shelton, K. H. \& Harold, G. T., (2008). Pathways between interparental conflict and adolescent psychological adjustment bridging links through children's cognitive appraisals and coping strategies. Journal of Early Adolescence, 28(4), 555582.

Silva, D. F. M. \& Hutz, C. S. (2002). Abuso infantil e comportamento delinqüente na adolescência: prevenção e intervenção. Em: HUTZ, C. S. (Org.). Situações de risco e vulnerabilidade na infância e na adolescência: Aspectos teóricos e estratégias de intervenção (pp. 151-185). São Paulo: Casa do Psicólogo.

Silvares, E. F. M. \& Souza, C. L. (2008). Discórdia conjugal: distúrbios psicológicos infantis e avaliação diagnóstica comportamental-cognitiva. Psicologia: Teoria e Prática, 10 (1), 200-213.

Skeer, M., McCormick, M. C., Normand, S. T., Buka, S. L. \& Gilman, S. E. (2009). A prospective study of familial conflict, psychological stress, and the development of substance use disorders in adolescence. Drug and Alcohol Dependence, 104, 6572 .

Sourander, A., Niemela, S., Santalahti, I., Helenius, H. \& Piha, J. (2008). Changes in psychiatric problems and service use among 8-year-old children: a 16year population-based time-trend study. Journal of the American Academy of Child and Adolescent Psycbiatry, 47(3),317-27.

Spell, A. W., Kelley, M. L., Wang, J., Self-Brown, S., Davidson, K. L., Pellegrin, A., Palcic, J. L., Meyer, K., Paasch, V. \& Baumeister, A. (2008). The moderating effects of maternal psychopathology on children's adjustment post-Hurricane Katrina. Journal of Clinical Child \& Adolescent Psychology, 37(3), 553-563.

Spruijt, E. \& Duindam, V. (2005). Problem behavior of boys and young men after parental divorce in the netherlands. Journal of Divorce \& Remarriage, 43(3), 141-155.

Stark, K. D., Humphrey, L. L., Crook, K. \& Lewis, K. (1990). Perceived family environments of depressed and anxious children: child's and maternal figure's perspectives. Journal of Abnormal Child Psychology, 18, 527-547.

Steck, B., Amsler, F., Grether, A., Dillier, A. S., Baldus, C., Haagen, M., Diareme, L., Tsiantis, J., Kappos, L., Bürgin, D. \& Romer, G. (2007). Mental health problems in children of somatically ill parents, e.g. multiple sclerosis. European Child \& Adolescent Psycbiatry, 16(3), 199-207.

Steinhausen, H., Haslimeier, C. \& Metzke, C. W. (2007). Psychosocial factors in adolescent and young adult self-reported depressive symptoms:
Causal or correlational associations? Journal of Youth \& Adolescence, 36, 89-100.

Sternberg, K. J., Lamb, M.E., Guterman, E. \& Abbott, C.B. (2006). Effects of early and later family violence on children's behavior problems and depression: a longitudinal, multi-informant perspective. Child Abuse \& Neglect, 30, 283-306.

Stocker, C. M., Richmond, M. K. \& Rhoades, G. K. (2007). Family emotional processes and adolescents' adjustment. Social Development, 16(2), 310-325.

Storch, E. A., Geffken, G. R., Merlo, L. J., Jacob, M. L., Murphy, T. K., Goodman, W. K., Larson, M. J., Fernandez, M. \& Grabill, K. (2007). Family accommodation in pediatric obsessive-compulsive disorder. Journal of Clinical Child and Adolescent Psychology, 36(2), 207-216.

Teodoro, M. L. M., Cardoso, B. M. \& Freitas, A. C. H. (2009). Afetividade e conflito familiar e sua relação com a depressão em crianças e adolescentes. Psicologia: Reflexão e Crítica, 23(2), 324-333.

Thompson-Brenner, H., Eddy, K. T., Satir, D. A., Boisseau, C. L. \& Westen, D. (2008). Personality subtypes in adolescents with eating disorders: aalidation of a classification approach. Journal of Child Psychology and Psychiatry, 49(2), 170-180.

Tonge, B. J., Hughes, G. C., Pullen, J. M., Beaufoy, J. \& Gold, S. (2008). Comprehensive description of adolescents admitted to a public psychiatric inpatient unit and their families. Australian and New Zealand Journal of Psychiatry, 42, 627-635.

Trudeau, L., Spoth, R., Randall, G. K. \& Azevedo, K. (2007). Longitudinal effects of a universal familyfocused intervention on growth patterns of adolescent internalizing symptoms and polysubstance use: gender comparisons. Journal of Youth \& Adolescence, 36, 725-740.

Van der Toorn, S. L. M., Huizink, A. C., Utens, E. M. W. J., Verhulst, F. C., Johan Ormel, J. \& Ferdinand, R. F. (2010). Maternal depressive symptoms, and not anxiety symptoms, are associated with positive mother-child reporting discrepancies of internalizing problems in children: a report on the TRAILS Study. European Child \& Adolescent Psychiatry, 19(4), 379-388.

VanderValk, I., Spruijt, E., Goede, M., Maas, C. \& Meeus, W. (2005). Family structure and problem behavior of adolescents and young adults: A growth-curve study. Journal of Youth and Adolescence, 34(6), 533-546.

Vegt, E. J. M., Ende, J., Ferdinand, R. F., Verhulst, F. C. \& Tiemeier H. (2009). Early childhood adversities and trajectories of psychiatric problems 
in adoptees: evidence for long lasting effects. Journal of Abnorm Child Psycholy, 37, 239-249.

Wagner, A., Falcke, D., Silveira, L. M. B. O. \& Mosmann, C. P. (2002). A comunicação em famílias com filhos adolescentes. Psicologia em Estudo, 7, 75-80.

Wight, R. G., Botticello, A. L. \& Aneshensel, C. S. (2006). Socioeconomic context, social support, and adolescent mental health: a multilevel investigation. Journal of Youth and Adolescence, 35(1), 115-126.

Windle, M., Brener, N., Cuccaro, P., Dittus, P., Kanouse, D. E., Murray, N., Wallander, J. \&
Schuster, M. A. (2010). Parenting predictors of early-adolescents' health behaviors: simultaneous group comparisons across sex and ethnic groups. Journal of Youth and Adolescence, 39(6), 594-606.

Zelkowitz, P., Paris, J., Guzder, J., Feldman, R., Roy, C. \& Rosval, L. (2007). A five-year follow-up of patients with borderline pathology of childhood. Journal of Personality Disorders, 21(6), 664-674.

Recebido em 10/10/2011

Reformulado em 27/11/2012

Aprovado em 14/02/2013

Sobre as autoras:

Adriana Raquel Binsfeld Hess é psicóloga pela Universidade do Vale do Rio dos Sinos UNISINOS (2008) e mestre em Psicologia Clínica (2010), com bolsa CAPES, pela mesma instituição. Atualmente cursa Doutorado em Psicologia na Universidade Federal do Rio Grande do Sul - UFRGS e é professora do Curso de Graduação em Psicologia das Faculdades Integradas de Taquara (FACCAT - Universidade Federal do Rio Grande do Sul, Porto Alegre/RS).

Denise Falcke é psicóloga pela Pontifícia Universidade Católica do Rio Grande do Sul (1996), com especialização em Terapia de Casal e Família pelo Instituto de Terapia Integradas (ITI), Mestrado em Psicologia Clínica (1998) e Doutorado em Psicologia pela Pontifícia Universidade Católica do Rio Grande do Sul (2003). Atualmente é professora e coordenadora do Programa de Pós-Graduação em Psicologia da Universidade do Vale do Rio dos Sinos (UNISINOS).

Contato com as autoras:

Faculdades Integradas de Taquara (FACCAT) - Curso de Psicologia

Adriana Raquel Binsfeld Hess

Rua Oscar Martins Rangel, 4500 (RS 115) - CEP 95600-000 - Taquara-RS, Brasil

E-mail: hess.adrianabinsfeld@gmail.com 